\title{
Factoring and Fourier transformation with a Mach-Zehnder interferometer
}

\author{
Johann Summhammer 円 \\ Atominstitut \\ Schuettelstr. 115, A-1020 Vienna, Austria
}

\begin{abstract}
The scheme of Clauser and Dowling (Phys. Rev. A 53, 4587 (1996)) for factoring $N$ by means of an $N$-slit interference experiment is translated into an experiment with a single Mach-Zehnder interferometer. With dispersive phase shifters the ratio of coherence length to wavelength limits the numbers that can be factored. A conservative estimate permits $N \approx 10^{7}$. It is furthermore shown, that sine and cosine Fourier coefficients of a real periodic function can be obtained with such an interferometer.
\end{abstract}

PACS: 03.65.Bz, 06.50.Mk, 07.60.Ly

Recently Clauser and Dowling (CD) have shown that factors of an integer $N$ can be determined by simply measuring the peaks of the intensity distribution on the screen behind a Young's $N$-slit arrangement [1]. This device is distinct from the currently much debated proposals for quantum computation, because it does not need the entanglement of several quantal degrees of freedom. Therefore it is very immune against decoherence and could readily be implemented with present technology. The drawback is that it will not exhibit the potential increase in computational power characteristic of entanglement. Nevertheless, the work of CD indicates that single particle interference arrangements have useful applications beyond physical measurements.

The purpose of this note is to point out that the CD-proposal can be translated into an experiment with only a single Mach-Zehnder interferometer. This will enhance the flexibility of this calculational device. A further point is that a Mach-Zehnder interferomter can also perform other computations, in particular cosine and sine Fourier transformations.

Let us first focus on the work of CD. This proposal shows, that in a suitably chosen central region on the detection screen behind an $N$-slit arrangement, all intensity peaks are equal if, and only if, the quantity $n \equiv \lambda R / a^{2}$ is a factor of $N$, where $\lambda$ is the wavelength of the incident radiation, $a$ is the center-to-center distance of the slits, and $R$ is the distance between the slits and the screen. Furthermore, the Fraunhofer limit is assumed $(R \gg a \gg \lambda)$. Different values of $n$ can be tested by adjusting any of the parameters, for instance $\lambda$. The probability amplitude caused by slit $i$ at a point $\mathbf{r}$ on the screen is given by $\psi_{i}(\mathbf{r})$. The probability that the particle will hit this point is therefore

$$
I(\mathbf{r})=\left|\sum_{i=1}^{N} \psi_{i}(\mathbf{r})\right|^{2}=\sum_{i=1}^{N}\left|\psi_{i}(\mathbf{r})\right|^{2}+2 \sum_{i=1}^{N-1} \sum_{j=i+1}^{N} \operatorname{Re}\left[\psi_{i}(\mathbf{r}) \psi_{j}^{*}(\mathbf{r})\right] .
$$

For the idea to be presented here, it is important to notice that the essential properties of $I(\mathbf{r})$ can also be obtained in measurements with a series of different two-slit experiments. This is so, because quantum mechanical probabilities are the second power of probability

\footnotetext{
${ }^{1}$ e-mail: summhammer@ati.ac.at
} 
amplitudes. Therefore, the interference term of $I(\mathbf{r})$, which is the double summation on the right hand side, only contains products made up of just two factors. And this feature is already present in the two-slit experiment. (This would not be the case, if, say, probabilities were the third power of probability amplitudes. Then the paradigm of quantum physics would only be fully contained in a three slit experiment.) The analytic behavior of the interference term of the $N$-slit experiment can therefore be recaptured, when adding the intensities of all permutations of two-slit experiments obtainable by covering $N-2$ of the $N$ slits. This procedure will not reproduce the behavior of the mean intensity. But we can neglect the mean intensity here, because it is only of statistical relevance.

Instead of doing several two-slit experiments with different distances of the slits, it is of course more convenient to use a Mach-Zehnder interferometer and vary the phase shift. A further advantage of a Mach-Zehnder interferometer is that it has only two outputs, instead of the continuum of a screen. Then, with an arrangement as shown in Fig.1, factoring can be achieved in the following way: The phase shift $\chi$ is increased in discrete steps $2 \pi / n$, such that $\chi_{j}=2 \pi j / n$. For simplicity we assume that at each step one particle is sent into the interferometer. We look at the outputs only when $j=k N, k=1,2,3 \ldots$. So at the $k^{\text {th }}$ observation the probability to register the particle at detector $A$ is

$$
p_{A}(k)=\frac{1}{2}\left[1+\cos \left(\frac{2 \pi k N}{n}\right)\right] .
$$

We have $p_{A}(k)=1$ only if $n$ is a factor of N. Suppose we perform $n$ observations, starting with $k=1$ until $k=n$. Then the sum of the particles registered in detector $A$ will be

$$
I_{n}=\sum_{k=1}^{n} p_{A}(k)=\frac{n}{2}+\frac{1}{2} \sum_{k=1}^{n} \cos \left(\frac{2 \pi k N}{n}\right) .
$$

For $n$ a factor of $N$ we have $I_{n}=n$, because then the phases in the cosine terms will all be multiples of $2 \pi$. However, if $n$ is not a factor, the cosine-terms of the summation will roughly cancel each other, and we will only have $I_{n} \approx n / 2$. To see this more clearly, we write $N / n=M+r$, where $M$ is the largest integer for which $N / n>M$, and $r$ is the rational remainder, $r=L / n$, with $L \in[1,2, \ldots, n-1]$. Then the summation over the cosine terms can be written as

$$
\sum_{k=1}^{n} \cos \left(\frac{2 \pi L}{n} k\right) \text {. }
$$

The phases of the cosines are now equidistantly spread in $n$ steps from the value $2 \pi L / n$, which is less than $2 \pi$, to $2 \pi L$, which is at most $2 \pi(n-1)$, so that when reducing the phases to the interval $[0,2 \pi]$, this interval will be used quite evenly.

For practical applications it is important to know what calculations are possible. Since the best interferometers today are operated with light, it is sufficient to look at the characteristics of such interferometers. If we implement the phase shift in the usual manner by increasing the path length difference of the two arms of the interferometer, the coherence length of the incident radiation sets the limit to the largest number $N$ that can be factored. According to eq.(3) the maximum phase shift to be set in this scheme is $2 \pi N$. Assuming a wavelength of $\lambda=500 \mathrm{~nm}$ and conservatively limiting the coherence length, given by $C=\lambda^{2} / \Delta \lambda$, where $\Delta \lambda$ is the standard deviation of the wavelength distribution, to $C=5 m$, numbers up to $N \approx 10^{7}$ can be factored. 
One also wants to know how long a calculation will take. When testing whether $n$ is a factor of $N$, one must set $n N$ different phase shifts. Since the largest factor to be checked is of the order of $\sqrt{N}$, the longest check will take a time proportional to $N^{3 / 2}$.

A further consideration is the maximum deviation permissible in the phase increments $2 \pi / n$, in order to ensure correct identification of the factors of $N$. Suppose the actual increment is $2 \pi /(n+d)$, where $d$ is the deviation. In the ideal case we have $d=0$, and when $n$ is a factor of $N$ the sum (3) yields an intensity of $n$. Now this sum will still be substantially larger than $n / 2$ (which would indicate a non-factor), if the deviation $d$ is such, that for the largest phase shift to be set (at $k=n$ ) we have $2 \pi N-\frac{\pi}{2}$ rather than the ideal value $2 \pi N$. Then the contribution of the last term of the sum is zero, whereas all the others are still positive, so that the total intensity will be roughly $n\left(\frac{1}{2}+\frac{1}{\pi}\right)$. This limits the permissible relative deviation of the phase shift increments from their ideal value to $\left|\frac{d}{n}\right| \leq(4 N)^{-1}$. For our example of above this would mean $\left|\frac{d}{n}\right| \leq 2.5 \times 10^{-8}$. Such accuracy can be achieved in optical interferometers, when the lengthening of one arm relative to the other is itself controlled interferometrically.

It is also possible to implement a kind of parallel computation with a setup as illustrated in Fig.2. Here, the numbers $n_{1}, n_{2}, \ldots, n_{7}$ can almost simultaneously be checked for being factors of $N$, thereby utilizing an incident particle for more than just one computation. The phase shifters in the various loops are simultaneously stepped up, but at the different increments $2 \pi / n_{1}, 2 \pi / n_{2}, \ldots, 2 \pi / n_{7}$, respectively. Let us assume $n_{i}<n_{j}$ for $i<j$. And for the sake of convenience we sum the intensity in a given detector as needed for testing for the largest of the possible factors of the loops involved. For instance, at detector $A$ (and also at $B$ ) we would sum until $k=n_{4}$, thus

$$
I_{A}=\sum_{k=1}^{n_{4}} \frac{1}{8}\left[1+\cos \left(\frac{2 \pi k N}{n_{1}}\right)\right]\left[1+\cos \left(\frac{2 \pi k N}{n_{2}}\right)\right]\left[1+\cos \left(\frac{2 \pi k N}{n_{4}}\right)\right] .
$$

The expectation values for $I_{A}, I_{B}$ and $I_{C}+I_{D}$ (the latter also just summed up to $n_{4}$ ) for the various possibilities are shown in Table I. Clearly, the results permit unique identification of the eight possible answers of interest.

Table I. Dependance of intensities on property of "factor" or "non-factor". Factors are indicated by "F", non-factors by "-". Intensities in units of $n_{4} / 8$.

\begin{tabular}{|c|c|c||c|c|c|}
\hline$n_{1}$ & $n_{2}$ & $n_{4}$ & $I_{A}$ & $I_{B}$ & $I_{C}+I_{D}$ \\
\hline \hline $\mathrm{F}$ & $\mathrm{F}$ & $\mathrm{F}$ & 8 & 0 & 0 \\
\hline $\mathrm{F}$ & $\mathrm{F}$ & - & 4 & 4 & 0 \\
\hline $\mathrm{F}$ & - & $\mathrm{F}$ & 4 & 0 & 4 \\
\hline $\mathrm{F}$ & - & - & 2 & 2 & 4 \\
\hline- & $\mathrm{F}$ & $\mathrm{F}$ & 4 & 0 & 0 \\
\hline- & $\mathrm{F}$ & - & 2 & 2 & 0 \\
\hline- & - & $\mathrm{F}$ & 2 & 0 & 2 \\
\hline- & - & - & 1 & 1 & 2 \\
\hline
\end{tabular}

The situation is a little bit more complicated for the interferometer loops following the lower arm of the $n_{1}$-interferometer. We must account for the possibility that $n_{1}$ is a factor of $N$. If we then measured the intensity at any of the detectors $E$ to $H$ after every $N$ 
phase increments, we would never detect a particle. Therefore it is necessary to start the loops on the lower arm of $n_{1}$ with a delay of around $n_{1} / 2$ increments. Similar arguments apply to any of the interferometers connected to an output which is dark when the phase shift is a multiple of $2 \pi$. For instance, testing whether $n_{3}$ and $n_{6}$ are factors of $N$ requires that one waits for about $\left(n_{1}+n_{3}\right) / 2$ increments, and from then on sums the intensities at $E$ (or at $F$ ) after every $N$ further increments. Then the expectation values for $I_{E}$ for the eight possibilities (i.e., which of $n_{1}, n_{3}, n_{6}$ is a factor of $N$ ) are analogous to those shown for $I_{A}$ in Table I. However, the offset relative to the total number of phase increments to be gone through is small, such that one can still speak of essentially parallel computation. But, of course, as this is a device not exploiting the entanglement of quantum systems, the number of loops, or the total amount of time, needed for factoring $N$ rises polynomially with $N$, rather than just logarithmically.

Let us now turn to how Fourier transformations can be performed by means of an interferometer. Specifically, it is possible to obtain the cosine and sine Fourier coefficients of a real valued positive function $f(t)$, which is periodic with the period $\tau$, and where $t$ denotes time. For this it is necessary that the intensity incident on the interferometer varies in time proportional to $f(t)$, and that the phase shift $\chi$ applied between the two paths of the interferometer increases linearly with time, e.g.

$$
\chi(t)=\frac{2 \pi m t}{\tau}
$$

where $m$ is an integer. In order to avoid problems of the change of energy of the particles when experiencing a time-dependent phase shift, we limit ourselves to slow rates, $m / \tau \ll$ $E / h$, where $E$ is the energy of the particles and $h$ is Planck's constant. Then the difference of the intensities at detectors $A$ and $B$ integrated over one period $\tau$ is

$$
I_{A}-I_{B}=c \int_{0}^{\tau} f(t) \cos \left(\frac{2 \pi m t}{\tau}\right) d t
$$

where $c$ is the proportionality constant between $f(t)$ and the incident intensity. As can be seen, $I_{A}-I_{B}$ is proportional to the $m^{\text {th }}$ cosine Fourier coefficient of $f(t)$. In order to obtain the sine Fourier coefficients, one only needs to add a constant phase shift of $-\pi / 2$ to $\chi(t)$.

Note that this calculational procedure is different from the experimental method employed in Fourier spectroscopy, which has been used in astronomy since the days of Michelson and which has recently also been used in electron [2] and neutron [3] interferometry. In Fourier spectroscopy one can measure the spectral distribution of the incident radiation, if this distribution is symmetric about the mean, but one gets no phase information.

\section{References}

[1] John F. Clauser, Jonathan P. Dowling, Phys. Rev. A 53, 4587 (1996). A detailed analysis of the intensity patterns produced by $N$-slit diffraction, with references to the original literature of classical optics, is given in J. F. Clauser and M. W. Reinsch, Appl. Phys. B 54, 380 (1992).

[2] F. Hasselbach, A. Schäfer, H. Wachendorfer, Nucl. Instr. Meth. A 363,232 (1995). 
[3] H. Rauch, H. Wölwitsch, H. Kaiser, R. Clothier and S. A. Werner, Phys. Rev. A 53, 902 (1996).

\section{Figure Captions}

Fig.1: Ideal Mach-Zehnder interferometer with semitransparent mirrors for beam splitting at the entrance and beam superposition at the exit. A phase shifter introduces the additional phase $\chi$ between the two paths. Particles are registered in detectors $A$ and $B$.

Fig.2: Series connection of several Mach-Zehnder interferometers for almost simultaneous determination whether the integers $n_{1}, \ldots, n_{7}$ are factors of $N$. The particles are registered at detectors $A$ to $H$. 


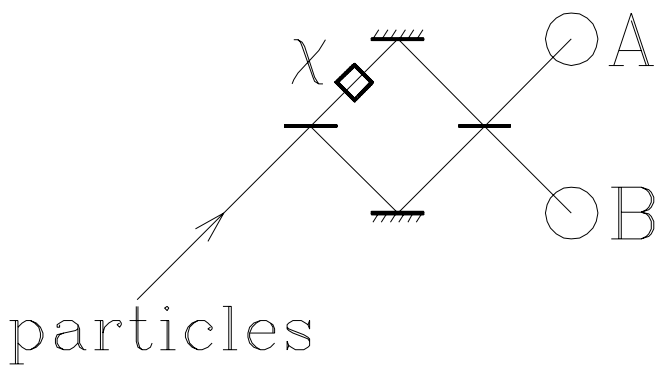




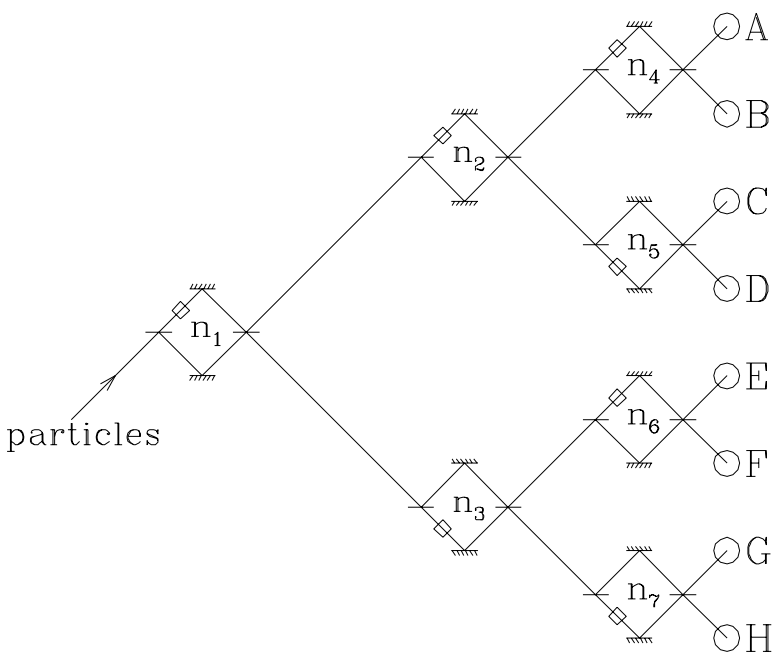

